\title{
Study of the Impact of Non-linear Piezoelectric Constants on the Acoustic Wave Propagation on Lithium Niobate
}

\author{
C. Soumali*, D. Benatia ${ }^{\dagger}$ \\ Laboratoire d'Electronique Avancée (L.E.A), Department of Electronics, University of Batna, Algeria
}

(Received 04 February 2016; revised manuscript received 13 June 2016; published online 21 June 2016)

\begin{abstract}
Impact of nonlinear piezoelectric constants on surface acoustic wave propagation on a piezoelectric substrate is investigated in this work. Propagation of acoustic wave propagation under uniform stress is analyzed; the wave equation is obtained by incorporating the applied uniform stress in the equation of motion and taking account of the set of linear and nonlinear piezoelectric constants. A new method of separation between the different modes of propagation is proposed regarding the attenuation coefficients and not to the displacement vectors. Detail calculations and simulations have made for Lithium Niobate $\left(\mathrm{LiNbO}_{3}\right)$; transformations between modes of propagation, under uniform stress, have been found. These results leads to conclusion that nonlinear terms affect the acoustic wave propagation and also we can make controllable acoustic devices.
\end{abstract}

Keywords: Surface acoustic wave, Piezoelectric substrate, Nonlinear material constants, Uniform stress, Attenuation coefficients.

DOI: $10.21272 /$ jnep.8(2).02013

PACS numbers: 43.25.Fe, 77.65.Dq,77.65. - j

\section{INTRODUCTION}

Lithium niobate $\left(\mathrm{LiNbO}_{3}\right)$ has attracted much interest as a piezoelectric substrate for fabrication of acousto-electronic devices such as delay lines, filters, temperature sensors and pressure sensors[1], etc. Recent development showed that the application of static biasing fields electric or mechanical was used to control the performances of the acousto-electronic devices [2-3].

The presence of biasing fields makes the piezoelectric apparently behave like a different substrate, and renders the linear theory of piezoelectricity invalid; the theory for infinitesimal incremental fields on finite biasing fields is used, which is a consequence of the nonlinear theory of electro-elasticity [4]. The nonlinear constitutive equations are linearized involving decomposition of the stress and the other field quantities into two parts, static biasing one, and the dynamic connected to the acoustic wave[5].

In this paper, the wave equation is obtained by using the linearized constitutive equations and incorporating the stress in the equation of motion and taking account of the published set linear and nonlinear materiel constants. A new method is proposed to select and separate between the different modes of propagation of acoustic waves, by regarding only to the attenuations coefficients. Numerical simulations and results for Lithium Niobate are presented.

\section{ANALYSIS OF ACOUSTIC WAVE PROPAGA- TION ON PIEZOELECTRIC SUBSTARTE UN- DER ACTION OF UNOFORM STRESS}

\subsection{Notations and Assumptions}

The coordinate system is shown in Fig. 1, the substrate occupies the half space $X_{3}<0$, the direction of propagation is along the axis $X_{1}$, the term representing the temporal variation is omitted and considering the anisotropy of the substrate used the notation of the tensors is used. The appearance of any index after a comma indicates that one must take the derivative of the quantity compared to the variable corresponding to the index. The derivative compared to time is indicated by a point on the variable to derive.

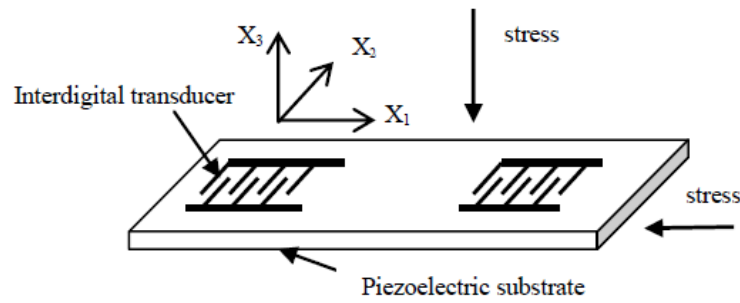

Fig. 1 - Piezoelectric structure of generation and detection of acoustic waves

The electric field is given by the gradient of an electrostatic potential and the magnetic field is neglected. The propagation velocity of electromagnetic waves is extremely high compared to that of elastic waves $\left(10^{5}\right)$, which justify the quasi static approximation. Other assumptions concerning the geometry of the device are necessary, the substrate is supposed semi-infinite, the electrodes are supposed perfect conductors and of null mass. It is convenient to suppose that there is not transverse variation i.e. according the direction $X_{2}$; the effect of diffraction is also neglected.

Also we suppose that the displacement related to the small wave generated by the digital transducer is separated from the displacement related to the biasing stress.

\subsection{Analysis}

Let us consider a small acoustic wave generated by an interdigital transducer (IDT) on a piezoelectric substrate,

\footnotetext{
* Soumali.chaabane@gmail.com

$\dagger$ dj_benatia@yahoo.fr
} 
propagating along $X_{1}$ and decaying in $X_{3}$ direction. Under application of uniform stress on the substrate:

The equation of motion can be written as following [6]:

$$
T_{i j, i}+\sigma_{i k} u_{j, k i}=\rho u_{j}
$$

$\rho$ : crystal density;

$T_{i j}$ : stress tensor;

$u_{i}$ : elastic displacement;

$\sigma_{l k}$ : initial stress present in the medium.

The constitutive equations for small acoustic wave superposed on biasing field are [5]:

$$
\begin{aligned}
& T_{i j}=C_{i j k l}^{*} S_{k l}-e_{k j i}^{*} E_{k} \\
& D_{i}=e_{i k l}^{*} S_{k l}+\varepsilon_{i k}^{*} E_{k}
\end{aligned}
$$

Where the new effective elastic, piezoelectric, dielectric constants are linear function of stress are defined as follows [7]:

$$
\begin{aligned}
& C_{a b k l}^{*}=C_{a b k l}^{E}+C_{a b k l q r}^{E} s_{q r m n} \sigma_{m n} \\
& e_{n a b}^{*}=e_{n a b}+e_{n a b k l} s_{k l m n} \sigma_{m n} \\
& \varepsilon_{m n}^{*}=\varepsilon_{m n}+H_{n m a b} s_{a b k l l} \sigma_{k l}
\end{aligned}
$$

$\sigma$. Stress tensor applied to the substrate $C_{a b k l q r}$ : Third-order nonlinear elastic tensor $e_{\text {nabkl: }}$ Third-order nonlinear piezoelectric tensor

$H_{n m a b}$ : Electrostrictive tensor

$C_{a b k l}$ : Second-order elastic tensor

$e_{\text {nab }}$ : Second-order piezoelectric tensor

$\varepsilon_{n m}$ : Second-order dielectric tensor

sklmn: Second-order compliance tensor

\subsection{Wave Equation}

Referring to the assumptions already token in previous section, Maxwell equations reduces to:

$$
\begin{aligned}
& D_{i, i}=q \\
& E_{i}=-\phi_{, i}
\end{aligned}
$$

Where:

$D_{i}$ : Electric flux density

$E_{i}:$ Electric field

$\phi_{i}$ : Electrostatic potential

$q$ : Free charge density

We suppose that the elastic displacement $u_{i}$ related to the small acoustic wave is separated from the displacement caused by the biasing so we can write:

$$
S_{k l}=\frac{1}{2}\left(u_{k, l}+u_{l, k}\right)
$$

\section{$S_{i j}:$ Strain tensor}

The substitution of equations (1), (4) and (5) in equation (2), for an area without charge density gives the wave equation for the substrate :

$$
\begin{aligned}
& \sigma_{i k} u_{j, k i}+C_{i j k l}^{*} u_{k, l i}+\varepsilon_{k i j}^{*} \phi_{, k i}=\rho \ddot{u_{j}} \\
& e_{i k l}^{*} u_{k, l i}-\varepsilon_{i k}^{*} \phi_{, k i}=0
\end{aligned}
$$

These equations are valid in the substrate $\left(X_{3}<0\right)$, for the second half space $\left(X_{3}>0\right)$ all acoustic fields are null, so the wave equation reduces to Laplace equation:

$$
\phi_{, i i}=0
$$

\subsection{Resolution of Wave Equation}

The acoustic displacements and the electric potential $\varphi$ of the acoustic wave must decay into the substrate and vanish at infinity, so the solutions of equations (6) should have the next form:

$$
\begin{aligned}
& u_{i}=A_{i} e^{\left(-\alpha \omega \frac{x_{3}}{v}\right)} e^{j \omega\left(t-\frac{x_{1}}{v}\right)} \\
& \varphi=A_{4} e^{\left(-\alpha \omega \frac{x_{3}}{v}\right)} e^{j \omega\left(t-\frac{x_{1}}{v}\right)}
\end{aligned}
$$

$A_{i}:$ Amplitude of the wave

v. surface wave velocity

$\alpha$ : decay coefficient in the substrate

The substitution of equations (8) and (9) into equation (6) leads to next linear homogeneous equations for the variable.

$\left[\begin{array}{cccc}\Gamma_{11}+\rho v^{2} & \Gamma_{12} & \Gamma_{13} & \Gamma_{14} \\ \Gamma_{21} & \Gamma_{22}+\rho v^{2} & \Gamma_{23} & \Gamma_{24} \\ \Gamma_{31} & \Gamma_{32} & \Gamma_{33}+\rho v^{2} & \Gamma_{34} \\ \Gamma_{41} & \Gamma_{42} & \Gamma_{43} & \Gamma_{44}\end{array}\right]\left[\begin{array}{c}A_{1} \\ A_{2} \\ A_{3} \\ A_{4}\end{array}\right]=\left[\begin{array}{l}0 \\ 0 \\ 0 \\ 0\end{array}\right]$

Where :

$$
\begin{gathered}
\Gamma_{11}=\left(C_{55}^{*}+\sigma_{33}\right) \alpha^{2}+\left(C_{15}^{*}+\sigma_{13}\right) 2 j \alpha-\left(C_{11}^{*}+\sigma_{11}\right) \\
\Gamma_{12}=\Gamma_{21}=C_{46}^{*} \alpha^{2}+\left(C_{14}^{*}+C_{56}^{*}\right) j \alpha-C_{16}^{*} \\
\Gamma_{13}=\Gamma_{31}=C_{35}^{*} \alpha^{2}+\left(C_{13}^{*}+C_{55}^{*}\right) j \alpha-C_{15}^{*} \\
\Gamma_{14}=\Gamma_{41}=e_{35}^{*} \alpha+\left(e_{15}^{*}+e_{31}^{*}\right) j \alpha-e_{11}^{*} \\
\Gamma_{22}=\left(\sigma_{33}+C_{44}^{*}\right) \alpha^{2}+\left(\sigma_{13}+C_{46}^{*}\right) 2 j \alpha-\left(C_{66}^{*}+\sigma_{11}\right) \\
\Gamma_{23}=\Gamma_{32}=C_{34}^{*} \alpha^{2}+\left(C_{36}^{*}+C_{45}^{*}\right) j \alpha-C_{56}^{*} \\
\Gamma_{24}=\Gamma_{42}=e_{34}^{*} \alpha^{2}+\left(e_{14}^{*}+e_{36}^{*}\right) j \alpha-e_{16}^{*} \\
\Gamma_{33}=\left(\sigma_{33}+C_{33}^{*}\right) \alpha^{2}+\left(\sigma_{13}+C_{35}^{*}\right) 2 j \alpha-\left(C_{55}^{*}+\sigma_{11}\right) \\
\Gamma_{34}=\Gamma_{43}=e_{33}^{*} \alpha^{2}+\left(e_{13}^{*}+e_{35}^{*}\right) j \alpha-e_{15}^{*} \\
\Gamma_{44}=-\varepsilon_{33}^{*} \alpha^{2}-2 j \alpha \varepsilon_{13}^{*}+\varepsilon_{11}^{*}
\end{gathered}
$$

In last equations the tensors $\mathrm{C}$, e and $\varepsilon$ are reduced to the standard matrix notation [8]

To avoid trivial solution of equation (10), the determinant of the left hand in equation (10) must be null. The development of this conditions leads to an eighth order polynomial in $\alpha$, which is equal to zero:

$$
\begin{aligned}
& C_{8} \alpha^{8}+j C_{7} \alpha^{7}+C_{6} \alpha^{6}+j C_{5} \alpha^{5}+C_{4} \alpha^{4}+j C_{3} \alpha^{3} \\
& +C_{2} \alpha^{2}+j C_{1} \alpha+C_{0}=0
\end{aligned}
$$


Where the coefficients are purely real for an assumed value of the speed.

\subsection{Selection Rule of the Valid Roots in the Sub- strate}

From the eight roots obtained, four only from roots are valid witch ensure decay in the substrate; these four roots represent four modes of propagation; a quasielectrostatic mode, a quasi-longitudinal mode and two quasi-transversal modes one fast and the other slow.

The roots have a complex form:

$$
\alpha=\alpha_{r}+j \alpha_{i}
$$

The first physic consideration is $\alpha_{r}$ must be positive to ensure decay into the substrate.

If $\alpha_{r}>0$ we have a surface acoustic wave (SAW);

If $\alpha_{r}=0$ we have a bulk acoustic wave (BAW) propagating in the direction $\theta$, where:

$$
\theta=\operatorname{arctg}\left(1 / \alpha_{i}\right)
$$

The electrostatic mode is characterized by no propagation so it is the mode the most attenuated; the longitudinal mode is characterized by direction of propagation parallel to $X_{1}$ however the two transversal modes are characterized by a direction perpendicular to $X_{1}$.

Taking account of all these physical considerations, we have suggested a rule to select and separate between the different modes in an algorithmic form regarding the decay coefficients only.

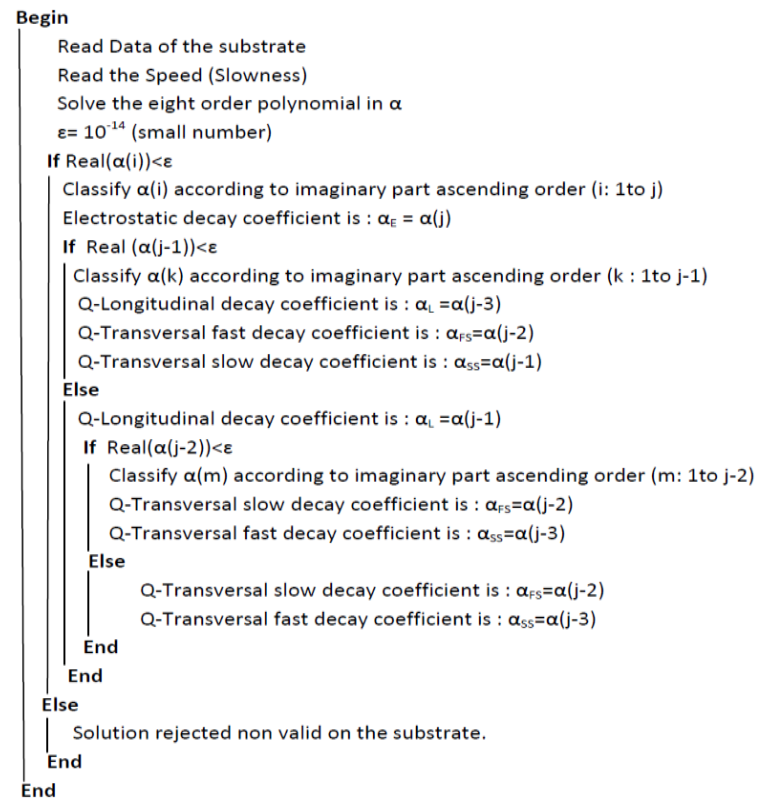

Algorithm 1 - Pseudo code for roots selection rule

\subsection{Displacement Vectors Calculus}

Once the decay coefficients are obtained and selected, one can express the total fields (mechanical displacement and electric potential), as a linear combination of the fields related to the selected decay coefficients so:

$$
\begin{aligned}
& u_{i}=\sum_{k=1}^{4} B_{k} A_{i}^{k} e^{\left(-\alpha_{k} \omega \frac{x_{3}}{v}\right)} e^{j \omega\left(t-\frac{x_{1}}{v}\right)} \\
& \varphi=\sum_{k=1}^{4} B_{k} A_{4}^{k} e^{\left(-\alpha_{k} \omega \frac{x_{3}}{v}\right)} e^{j \omega\left(t-\frac{x_{1}}{v}\right)}
\end{aligned}
$$

Where the coefficients are the proportions between the different modes. In order to evaluate these coefficients $\left(B_{k}\right)$ we have to use the next boundary conditions:

The crystal surface is stress free $\left(T_{3 j}=0\right.$ at $\left.X_{3}=0\right)$.

If a stress $\sigma$ is applied on the substrate, the total stress will be:

$$
T_{i j}^{\prime}=T_{i j}+\sigma_{i k} u_{j, k}
$$

The substitution of equations (12) and ((13) into equation (14) yields to the next set of homogeneous equations for the coefficients

$$
\begin{aligned}
& \sum_{k=1}^{4}\left\{A_{1 k}\left[j\left(\sigma_{31}+C_{15}^{*}\right)+\alpha_{k}\left(\sigma_{33}+C_{55}^{*}\right)\right]+\right. \\
& +A_{2 k}\left(j C_{56}^{*}+\alpha_{k} C_{45}^{*}+\right. \\
& \left.+A_{3 k}\left(j C_{55}^{*}+\alpha_{k} C_{35}^{k}\right)+A_{4 k}\left(j e_{15}^{*}+\alpha_{k} e_{35}^{*}\right)\right\} B_{k}=0 \\
& \sum_{k=1}^{4}\left\{A_{1 k}\left(j C_{14}^{*}+\alpha_{k} C_{45}^{*}\right)+\right. \\
& +A_{2 k}\left[j\left(\sigma_{13}+C_{46}^{*}\right)+\alpha_{k}\left(\sigma_{33}+C_{44}^{*}\right)\right]+ \\
& \left.+A_{3 k}\left(j C_{45}^{*}+\alpha_{k} C_{34}^{*}\right)+A_{4 k}\left(j e_{14}^{*}+\alpha_{k} e_{34}^{*}\right)\right\} B_{k}=0 \\
& \sum_{k=1}^{4}\left\{A_{1 k}\left(j C_{13}^{*}+\alpha_{k} C_{35}^{*}\right)+A_{2 k}\left(j C_{36}^{*}+\alpha_{k} C_{34}^{*}\right)+A_{3 k}\right. \\
& {\left[j\left(\sigma_{31}+C_{35}^{*}\right)+\alpha_{k}\left(\sigma_{33}+C_{33}^{*}\right)\right]+} \\
& \left.+A_{4 k}\left(j e_{13}^{*}+\alpha_{k} e_{33}^{*}\right)\right\} B_{k}=0
\end{aligned}
$$

In the above system of equations we have four variables $B_{k}$ and three equations only so we have to normalize by $B_{4}$ to obtain a solution, in this case the solutions are the eigenvectors of the system.

\section{SIMULATION AND RESULTS}

In this section we simulate the propagation of a small acoustic wave on Lithium Niobate subjected to uniaxial stress.

The application of uniform stress according the direction $\mathrm{X}_{1}$ changes the initial 23 point symmetry to orthorhombic class 222 according to the curie principle of symmetry [9]; as consequence the material constants are modified:

$$
\begin{aligned}
& C_{11}^{*}=C_{11}+\left[C_{111} s_{11}+\left(C_{112}+C_{113}\right) s_{12}\right] \sigma \\
& C_{33}^{*}=C_{11}+\left[C_{112} s_{11}+\left(C_{113}+C_{111}\right) s_{12}\right] \sigma \\
& C_{13}^{*}=C_{12}+\left[C_{113} s_{11}+\left(C_{123}+C_{112}\right) s_{12}\right] \sigma \\
& C_{44}^{*}=C_{44}+\left[C_{144} s_{11}+\left(C_{166}+C_{155}\right) s_{12}\right] \sigma \\
& C_{55}^{*}=C_{44}+\left[C_{155} s_{11}+\left(C_{144}+C_{166}\right) s_{12}\right] \sigma
\end{aligned}
$$




$$
\begin{aligned}
C_{66}^{*} & =C_{44}+\left[C_{166} s_{11}+\left(C_{155}+C_{144}\right) s_{12}\right] \sigma \\
C_{12}^{*} & =C_{12}+\left[C_{112} s_{11}+\left(C_{113}+C_{123}\right) s_{12}\right] \sigma \\
C_{23}^{*} & =C_{12}+\left[C_{123} s_{11}+\left(C_{112}+C_{113}\right) s_{12}\right] \sigma \\
C_{22}^{*} & =C_{11}+\left[C_{113} s_{11}+\left(C_{111}+C_{112}\right) s_{12}\right] \sigma \\
e_{14}^{*} & =e_{14}+\left[e_{114} s_{11}+\left(e_{124}+e_{134}\right) s_{12}\right] \sigma \\
e_{36}^{*} & =e_{14}+\left[e_{124} s_{11}+\left(e_{134}+e_{114}\right) s_{12}\right] \sigma \\
e_{25}^{*} & =e_{14}+\left[e_{134} s_{11}+\left(e_{114}+e_{124}\right) s_{22}\right] \sigma \\
\varepsilon_{11}^{*} & =\varepsilon_{11}+\left[H_{11} s_{11}+\left(H_{12}+H_{13}\right) s_{12}\right] \sigma \\
\varepsilon_{33}^{*} & =\varepsilon_{11}+\left[H_{11} s_{11}+\left(H_{31}+H_{32}\right) s_{12}\right] \sigma
\end{aligned}
$$

Taking in account equation (18) and the set of linear and non linear material constants[4]-[10]; the eight order polynomial in equation (11) is solved and the decay coefficients are carried out and classified in four modes of propagations according to the selection rule of valid roots already proposed in the previous section.

Table 1 - Simulation parameters and cutoff slownesses

\begin{tabular}{|l|l|l|}
\hline $\begin{array}{c}\text { Propagation } \\
\text { mode }\end{array}$ & Stress & Cutoff slowness \\
\hline Quasi- & $0 \mathrm{~Pa}$ & $1.3452 \mathrm{~s} / \mathrm{m}$ \\
Longitudinal & $1 \mathrm{MPa}$ & $1.5264 \mathrm{~s} / \mathrm{m}$ \\
& $100 \mathrm{MPa}$ & $1.4272 \mathrm{~s} / \mathrm{m}$ \\
& $1 \mathrm{GPa}$ & $1.5264 \mathrm{~s} / \mathrm{m}$ \\
\hline Fast Quasi- & $0 \mathrm{~Pa}$ & $2.7708 \mathrm{~s} / \mathrm{m}$ \\
Transversal & $1 \mathrm{MPa}$ & $3.0144 \mathrm{~s} / \mathrm{m}$ \\
& $100 \mathrm{MPa}$ & $3.0144 \mathrm{~s} / \mathrm{m}$ \\
& $1 \mathrm{GPa}$ & $3.0144 \mathrm{~s} / \mathrm{m}$ \\
\hline Slow Quasi- & $0 \mathrm{~Pa}$ & $2.8104 \mathrm{~s} / \mathrm{m}$ \\
Transversal & $1 \mathrm{MPa}$ & $2.5184 \mathrm{~s} / \mathrm{m}$ \\
& $100 \mathrm{MPa}$ & $2.5184 \mathrm{~s} / \mathrm{m}$ \\
& $1 \mathrm{GPa}$ & $2.6176 \mathrm{~s} / \mathrm{m}$ \\
\hline
\end{tabular}

Simulations done for Lithium niobate for the set of stress ( $1 \mathrm{MPa}, 100 \mathrm{MPa}, 1 \mathrm{GPa})$ shows a shift of cutoff slownesses as mentioned in Table 1 , witch result in a transformation of BAW mode of propagation to SAW mode of propagation in these intervals, also we remark that the imaginary part the decay coefficient of all modes is null above the cutoff slownesses witch result in the propagation of pure SAW mode.

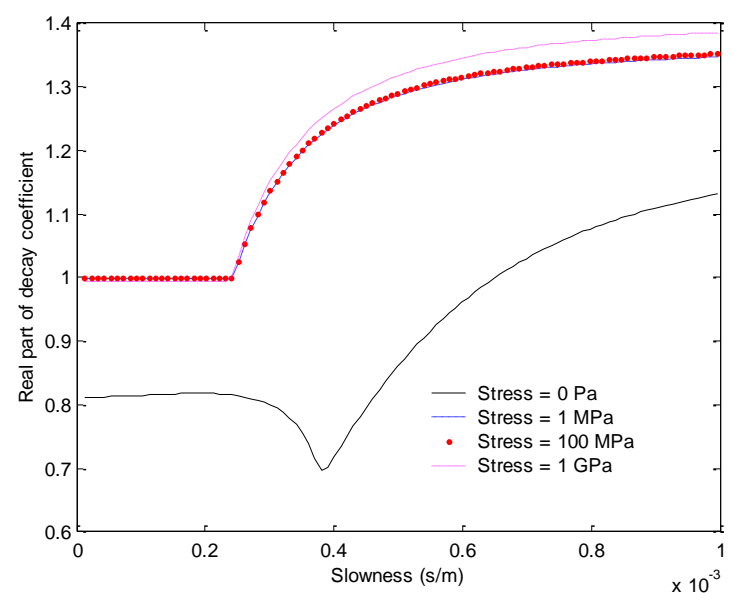

Fig. 2a - Real part of decay coefficient of quasi-electrostatic mode

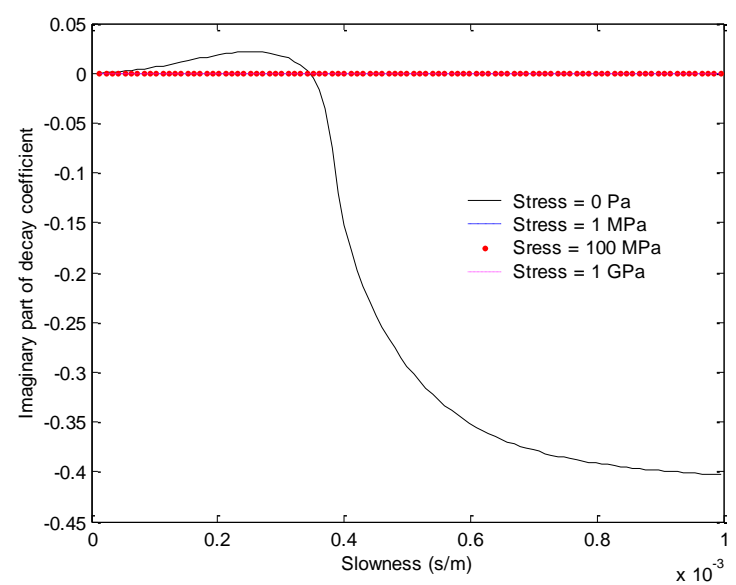

Fig. 2b-Imaginary part of decay coefficient of quasielectrostatic mode

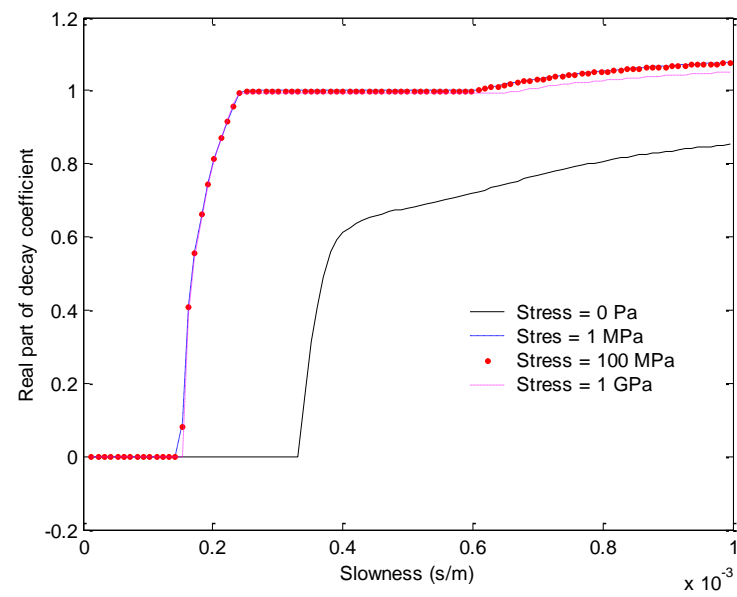

Fig. 3-Real part of decay coefficient of quasi-longitudinal mode

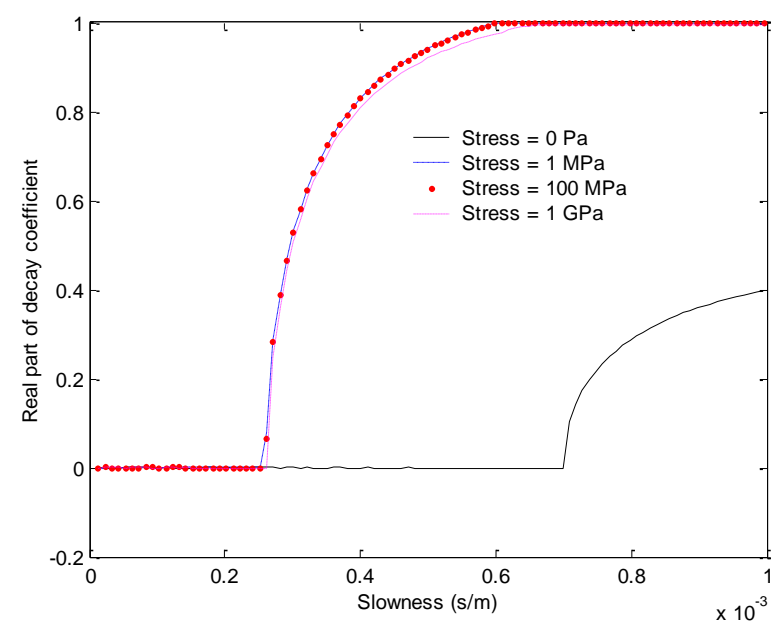

Fig. 4a-Real part of decay coefficient of slow quasitransversal mode 


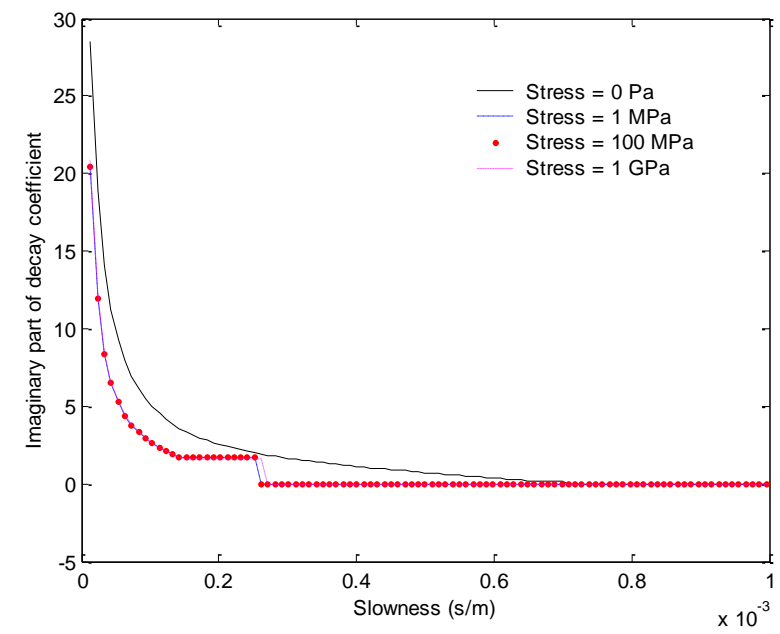

Fig. 4b-Imaginary part of decay coefficient of slow quasitransversal mode

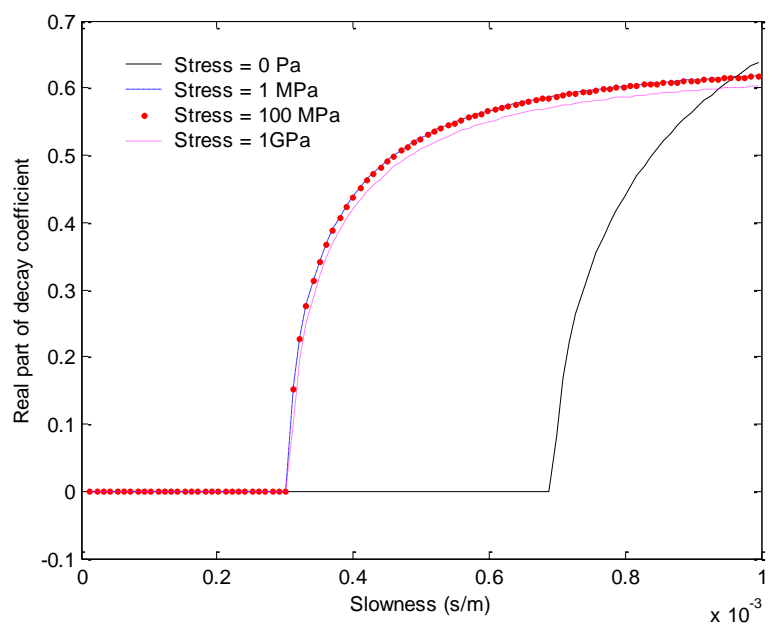

Fig. 5a-Real part of decay coefficient of fast quasitransversal mode

\section{REFERENCES}

1. J.C. Andle, J.F. Vetelino, Sensor. Actuat. A 44, 167 (1994).

2. F. Kubat, W. Ruile, T. Hesjedal, J. Stotz, L.M. Reindl, IEEE T. Ultrason. Ferroel. Freq. Control. 51 No 11, 1437 (2004).

3. Y. Jing, J. Chen, X. Gong, J. Duan, IEEE T. Ultrason. Ferroel. Freq. Contr. 54 No 5, 906 (2007).

4. J. Yang, An introduction to the theory of piezoelectricity (Springer: 2005)

5. D. Gafka, Archive. Acoustic. 19 No 2, 239 (1994).

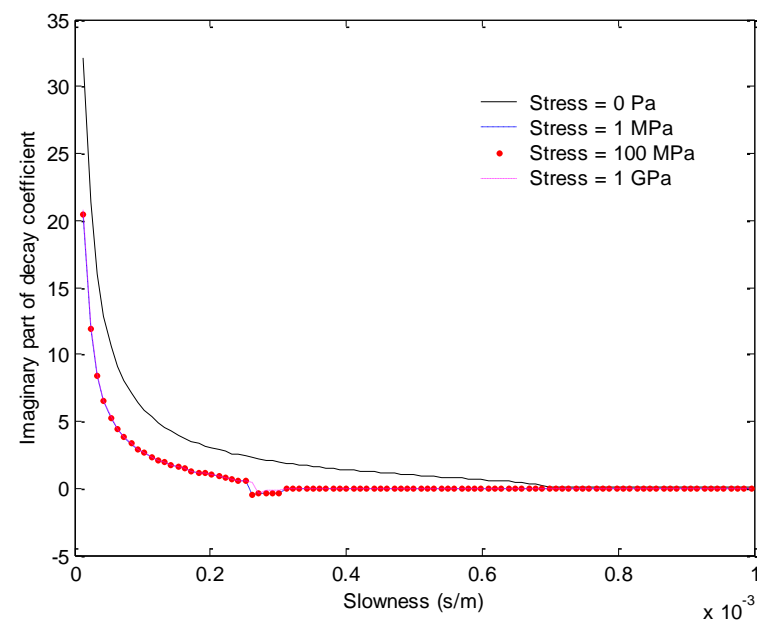

Fig. 5b- Imaginary part of decay coefficient of fast quasitransversal mode

\section{CONCLUSION}

Equations of propagation of a small acoustic waves generated by an interdigital transducer in a piezoelectric substrate subjected to an external stress are obtained by incorporating the stress in the equation of motion and taking account of the set of linear and nonlinear material constants. The effect of an external on the spectrum of acoustic waves is analyzed for several values of stress. Simulations shows that transformation of BAW mode to SAW mode occur for all the spectrum of acoustic waves (quasi-longitudinal, quasitransversal fast, quasi-transveral slow) under application of uniform stress. Also propagation of pure SAW mode have been detected above the new cutoff slownesses. These results can be useful for designing of controllable acousto-electronic devices such as delay lines, pressure sensors etc.

6. V.V. Bolotin, Nonconsevative problems of the theory of elastic stability (Macmillan: New York: 1963).

7. S.I. Burkov, O.P. Zolotova, B.P. Sorokin, P.P. Turchin, Ultrasonics 55, 104 (2015).

8. B.A. Auld, Acoustic fields and waves in solids (New York: Wiley: 1973).

9. S.I. Burkov, O.P. Zolotova, B.P. Sorokin, J. Siberian Federal University. Math. Phys. 7 No 1, 10 (2014). 